\title{
Measuring Staple Line Security and Compression Uniformity With an Advanced Endoscopic Powered Stapler
}

\author{
Austin Wise ${ }^{1}$, Jason Rector ${ }^{1}$, Katelyn Orr ${ }^{1}$, David W Singleton ${ }^{2, *}$, and Crystal D Ricketts ${ }^{2}$ \\ ${ }^{1}$ Research and Development, Ethicon, Inc., Blue Ash, Ohio, USA \\ ${ }^{2}$ Medical Affairs, Ethicon, Inc., Blue Ash, Ohio, USA \\ *Corresponding author: David W Singleton, Ph D, Medical Affairs, Ethicon, Inc., 4545 Creek Rd, Blue Ash, OH 45242, Ohio, USA, Tel: +1 (513) \\ 337-1027; E-mail: dsingl12@its.jnj.com
}

Received: 13 Feb, 2021 | Accepted: 09 Mar, 2021 | Published: 17 Mar, 2021

Citation: Wise A, Rector J, Orr K, Singleton DW, Ricketts CD (2021) Measuring Staple Line Security and Compression Uniformity With an Advanced Endoscopic Powered Stapler. J Surg Open Access 7(2): dx.doi.org/10.16966/2470-0991.233

Copyright: (c) 2021 Wise A, et al. This is an open-access article distributed under the terms of the Creative Commons Attribution License, which permits unrestricted use, distribution, and reproduction in any medium, provided the original author and source are credited.

\begin{abstract}
Objective: The purpose of this study was to evaluate the performance of a powered surgical stapler with a new anvil and motor design intended to improve the formation and security of staple lines.

Methods: Benchtop analyses of staple formation and leak testing were performed using ex vivo porcine stomach and ileum tissue, respectively. Device compression was assessed by firing the test device (ECHELON ${ }^{\mathrm{TM}}+$ Stapler with GST Reloads) and comparator stapling systems onto a controlled medium followed by analysis with laser microscopy and compression profile mapping. Evaluation of tissue transections with the test device were performed by 27 surgeons using simulated surgical models with excised porcine tissues, followed by completion of a post-procedure questionnaire.

Results: The test stapler exhibited a significantly higher mean staple cutline leak pressure $(93.6 \mathrm{mmHg})$ compared to the Signia ${ }^{\mathrm{TM}}$ Power Handle system and Endo GIA ${ }^{T M}$ Ultra Universal Standard Handle $(74.7 \mathrm{mmHg}$ and $61.9 \mathrm{mmHg}$, respectively). In addition, the number of stapled specimens that leaked at pressures less than or equal to 30 and $52 \mathrm{mmHg}$ was significantly lower for the test device. The frequency of malformed staples was $1.14 \%$ for the test device versus $4.28 \%$ for Signia and Endo GIA combined $p<0.001$. Compression testing revealed a median percent difference of $39.95 \%$ between the inner and outer staple row pressures for the test device, as compared to $62.28 \%$ for Signia and $57.84 \%$ for Endo GIA, p<0.001. Tissue transection times and tactile and/or audio feedback from the test device were rated as acceptable by all surgeons participating in the study.

Conclusion: The ECHELON+ Stapler with GST Reloads provided a lower rate of staple malformation, more uniform compression, and fewer staple line leaks compared to similar devices in benchtop analyses. Tissue transection times and feedback with the test device were determined to be adequate.
\end{abstract}

Keywords: Powered stapler; Staple malformation; Staple line leak; Compression; Hemostasis

\section{Introduction}

In minimally invasive procedures, surgical staplers are used to provide tissue approximation and hemostasis without causing ischemia or tearing of tissue. The creation of a secure staple line is dependent on multiple factors such as tissue compressibility and thickness, pre-firing compression (precompression), proper staple formation, correct staple height, minimal tissue movement during stapler application, and adequate stapler firing times [1-4]. Ideal prefiring compression force and duration are known to be dependent on the specific tissue's biomechanical properties and interstitial fluid content $[2,5]$. The goal of optimal compression is to provide a balance between secure hemostasis and adequate perfusion without the development of tissue shearing [2,6]. Several preclinical studies have demonstrated the influence that precompression and the stapler firing time can have on hemostasis and staple line leak rates [7-9]. Matsuzawa F, et al. [7] found that longer precompression and slower firing times with a powered stapler were associated with optimal staple formation and reduced occurrence of serosal lacerations in transected porcine stomach. Similarly, Nakayama S, et al. noted that sufficient precompression time was correlated with proper staple formation [8].

Tissue thickness is also an important consideration, as leaks may occur due to a mismatch between staple height and tissue thickness. To decrease the risk of staple line complications during the transection of thick tissues, it is important that the appropriate stapler cartridge selection is made [10]. If tissue is too thick for the staple height selected, ischemia or serosal shearing may occur, potentially leading to leakage or necrosis. On the other hand, if tissue is too thin for the selected closed staple height, then inadequate apposition of tissues may cause leaking, bleeding or dehiscence [2].

The ECHELON+ Stapler with GST Reloads (EPS) provides articulation for maneuverability and motorized/powered firing for reduced tip movement during stapling. The Gripping Surface Technology (GST), designed for enhanced tissue stabilization, has 
been associated with a decreased need for staple line interventions and fewer hemostatic complications compared to standard stapler cartridge reloads in sleeve gastrectomy procedures $[3,11]$. Reported benefits associated with powered staplers also include decreased hospital costs as well as improved clinical outcomes $[12,13]$. The objective of this study was to evaluate the new EPS stapler in benchtop testing for staple line leakage, frequency of staple malformation, and compression, as well as assessment of tissue transections in simulated surgical models.

\section{Methods}

\section{Endoscopic Staplers}

The test device in this study was the $60 \mathrm{~mm}$ ECHELON+ stapler (Figure 1) with newly designed Echelon+ anvil (Ethicon Inc.; Blue Ash, Ohio, USA), using $60 \mathrm{~mm}$ blue (GST60B), green (GST60G), and black (GST60T) staple cartridge reloads, for regular and thick tissue. For comparative analyses, the Endo GIA ${ }^{\text {mo }}$ Ultra Universal Standard Handle (EGIA) and Signia ${ }^{\text {mix }}$ Powered stapling System (Signia) with Tri-Staple ${ }^{\text {trut }}$ technology reloads (Medtronic PLC; Minneapolis, MN, USA) were tested in parallel with the EPS stapler. All devices were used in accordance with specific device instructions for use.

\section{Ex vivo leak testing}

To evaluate staple line leak pressures for EPS and comparator staplers, sections of excised porcine ileum were stapled and analyzed using a leak visualization fixture system. Briefly, fresh porcine ileum of $1.75-2.25 \mathrm{~mm}$ thickness was stapled by firing the stapler longitudinally along the tissue followed by attachment of the proximal end of the tissue staple line to a luer lock fitting securely tied with suture. The distal end of the stapled tissue was also tied off with suture to create a finished test sample specimen. For each stapler firing, specimens were defined as left or right according to which side of the cut line the tissue was from in relation to the device anvil. Each device was fired 15 times to produce 30 stapled porcine ileum specimens, including both the left and right sides. At the initial appearance of a leak, the pressure and leak type (cutline or staple leak) were recorded. If the initial leak type was a staple leak, the pressure was recorded as the initial leak pressure and the pressure was further increased until a cutline leak was observed. If the initial leak type was a cutline leak, the pressure was recorded as both the initial leak pressure and the cutline leak pressure. In addition, the total number of specimens that exhibited a leak of any type (staple or cutline) at pressures at or below 30 and $52 \mathrm{mmHg}$ was recorded. Evaluation of leak performance at $52 \mathrm{mmHg}$ was included as a high threshold, as it was twice the average leak test pressure of 26 mmHg noted in porcine colon [14]. This testing was performed for EPS, as well as the Medtronic EGIA and Signia systems.

Staple malformation analysis: For evaluation of malformed staples, devices were fired across freshly harvested porcine stomach.
All firings were articulated left or right. Staple lines were evaluated for staple formed quality using CT-scanning and data analysis with the North Star Imaging X50 system and efX-CT software (North Star Imaging, Minnesota, USA). Each staple was individually evaluated and classified according to closed formation. A malformed staple was defined as any staple having at least one leg that did not conform to the proper B shape. Tested devices included the EPS, EGIA, and the Signia stapler.

\section{Compression testing}

To evaluate the EPS stapler for uniformity of compression, devices were fired across a controlled medium followed by scanning of the completed staple lines with the Keyence VR-5000 One-Shot 3D Laser microscope and software (Keyence Corporation, Japan) for capture of compression profiles for each sample. Data were then processed by Kinetic Vision (Cincinnati, OH, USA) to translate compression to pressures. The compression/pressure values were analyzed for comparison of the test and control group devices. Measurements of compression at the outer and inner rows were taken at three locations along the staple line for both the right and left sides of the cut line for capturing variation on both sides of the device anvil. The difference in pressures between outer and inner stapler rows was determined at each location to yield a total of 6 data points per stapler firing, with 12 staple lines analyzed per device/reload combination, $(\mathrm{N}=72$ data points).

\section{Tissue transection assessment}

To collect data regarding tissue transection efficiency and tactile/ audio feedback with the EPS stapler, 27 surgeons used the device to complete steps of a simulated procedure in a simulated patient model with harvested porcine tissues. This analysis was conducted at two test locations in the USA (Cincinnati OH, and Chicago IL), and included surgeons from bariatric $(n=13)$, colorectal/general $(n=8)$, and thoracic $(n=6)$ specialties. Each surgeon performed transections on tissue types relevant to their specialty followed by assessment of the EPS device via completion of a questionnaire. None of the surgeons had received previous training with the test device prior to the study.

\section{Statistical methods}

For analysis of staple line leak pressures, two-sample t-tests were used for comparison of means. One-way ANOVA was employed to assess mean thicknesses of ileum tissue used for each device group. The number and frequency of specimens in which leaks occurred at or below defined pressures and the number of malformed staples were compared for device groups using two-sample tests of proportions. For comparison of compression medians, the Mann-Whitney test was used. An alpha level of significance was taken as 0.05 in all comparative analyses.

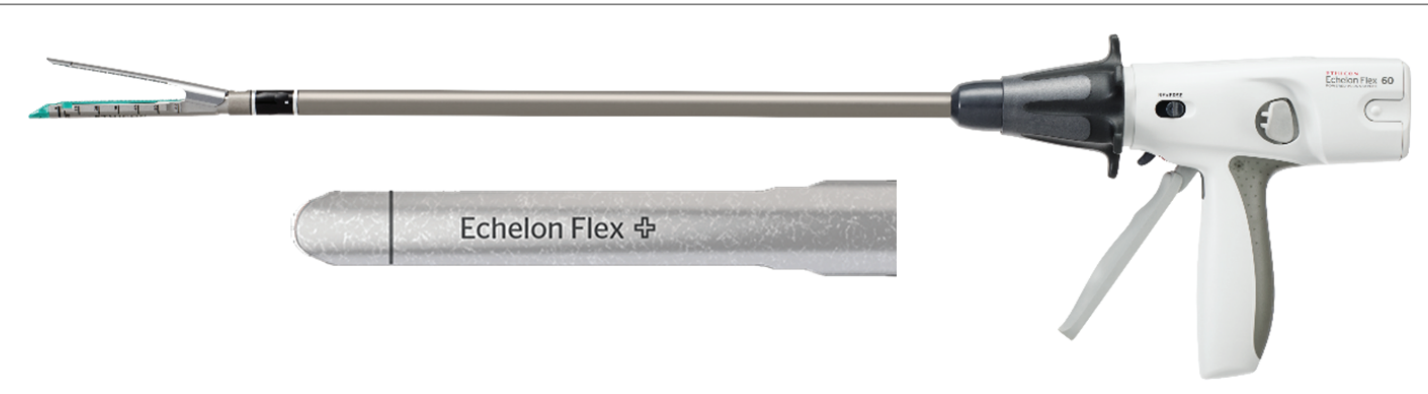

Figure 1: The ECHELON+ Stapler 


\section{Results}

\section{Staple line leak tests}

Table 1 lists the mean pressures at which initial leaks and specific cut edge (cutline) leaks were observed for the EPS stapler used with blue staple reload cartridges and the Signia and EGIA staplers with purple (EGIA60AMT) cartridge. The EPS exhibited higher initial and cutline leak pressures compared to Signia and EGIA staplers. In addition, significantly fewer leaks were seen with EPS relative to comparator staplers, and no leaks were noted for EPS at pressures of $30 \mathrm{mmHg}$ and below.

\section{Staple malformation}

Table 2 summarizes the analysis of staple malformation following firing of devices onto porcine stomach. The sample sizes included a minimum of 30 firings per each device/reload combination. For EPS staple lines using green and black cartridge reloads, the frequency of malformed staples was significantly lower compared to EGIA and Signia systems with purple and black cartridge reloads, $\mathrm{p} \leq 0.004$.

\section{Compression analysis}

Figure 2 shows staple line compression (pressure) maps for the EPS stapler with blue cartridge reload and EGIA using the purple (EGIA60AMT) Tri-Staple reload. The maps indicate more uniform compression for the EPS stapler relative to EGIA. Table 3 lists the median pressures for outer and inner staple rows, and the median percent difference in pressure between outer and inner staples for the EPS stapler using green and blue reloads, compared to Signia and EGIA staplers with purple cartridge reloads. The median percent difference was significantly lower for EPS compared to both Signia and EGIA staplers, $\mathrm{p}<0.001$ for all comparisons. EPS with green reloads alone, or blue reloads alone each also showed statistically lower percent differences across the outer and inner rows compared to Signia and EGIA, (data not shown).

\section{EPS transection questionnaire}

Table 4 summarizes the results of surgeon responses following transection of porcine tissues with the EPS stapler in simulated bariatric, colorectal, and thoracic surgical models. A rating of acceptable was documented by all 27 surgeons regarding the time necessary to complete the transections, and for tactile and/or audible feedback during the application of the device. There were no unacceptable ratings for any application of the EPS stapler.

\section{Discussion}

This study was undertaken to evaluate performance of the ECHELON+ stapler with GST reloads (EPS) in the context of a redesigned anvil and motor. Results indicated that the new device exhibited significantly fewer leaks at staple lines in ex vivo porcine ileum, compared to the Signia and EGIA staplers. In addition, the mean pressure at which staple line leaks occurred was higher for EPS compared to Signia, suggesting a more secure staple line with the EPS device. The frequency of malformed staples for EPS using green and black cartridge reloads for thick tissue (stomach) was significantly lower relative to both the EGIA and Signia staplers with purple and black reloads. The evaluation of tissue compression following stapling of a controlled medium with EPS and comparator devices indicated

Table 1: Staple line leak pressures for the Echelon+ stapler using blue GST reloads (EPS-GST60B), the Signia Power Handle with purple reload (SigniaEGIA60AMT), and EGIA with purple reloads (EGIA-EGIA60AMT). *Statistically significant compared to EPS, $p<0.05$.

\begin{tabular}{|l|c|c|c|}
\hline \multicolumn{1}{|c|}{ Variable } & EPS-GST60B & Signia-EGIA60AMT & EGIA-EGIA60AMT \\
\hline Initial leak pressure $(\mathrm{mmHg})$ mean $\pm \mathrm{SD}$ & $59.8 \pm 28.5(\mathrm{~N}=30)$ & $47.5 \pm 27.9(\mathrm{~N}=30)$ & $38.8^{*} \pm 21.2(\mathrm{~N}=30)$ \\
\hline Cutline leak pressure $(\mathrm{mmHg})$ mean $\pm \mathrm{SD}$ & $93.6 \pm 33.5(\mathrm{~N}=28)$ & $74.7^{*} \pm 31.9(\mathrm{~N}=29)$ & $61.9^{*} \pm 25.7(\mathrm{~N}=30)$ \\
\hline Number of leaked specimens at $\leq 30 \mathrm{~mm} \mathrm{Hg}$ & 0 & $7^{*}$ & $9^{*}$ \\
\hline Number of leaked specimens at $\leq 52 \mathrm{mmHg}$ & 14 & $23^{*}$ & $25^{*}$ \\
\hline
\end{tabular}

Table 2: Malformed staples in ex vivo porcine stomach stapled with: EPS/green reloads (GST60G) and black reloads (GST60T), EGIA with purple (EGIA60AMT) and black (EGIA60AXT) reload, and Signia using purple (EGIA60AMT) and black (SIG60AXT) reloads.

\begin{tabular}{|l|c|c|c|c|}
\hline \multicolumn{1}{|c|}{ Device/reloads } & Total Staples (N) & Malformed $\mathbf{n}$ (\%) & Difference (\%) vs. EPS & P-Value vs. EPS \\
\hline EPS/green and black & $\begin{array}{c}\mathrm{N}=5280 \\
\text { green=2640 } \\
\text { black=2640 }\end{array}$ & $60(1.14)$ & N/A & N/A \\
\hline EGIA/purple and black & $\begin{array}{c}\mathrm{N}=5940 \\
\text { purple=2970 } \\
\text { black=2970 }\end{array}$ & $406(6.84)$ & 5.70 & $<0.001$ \\
\hline Signia/purple and black & $\begin{array}{c}\mathrm{N}=5940 \\
\text { purple=2970 } \\
\text { black=2970 }\end{array}$ & $103(1.73)$ & 0.59 & $=0.004$ \\
\hline $\begin{array}{l}\mathrm{N}=11880 \\
\text { EGIA and Signia combined/ }=5940 \\
\text { purple and black }\end{array}$ & $509(4.28)$ & 3.14 & $<0.001$ \\
\hline
\end{tabular}



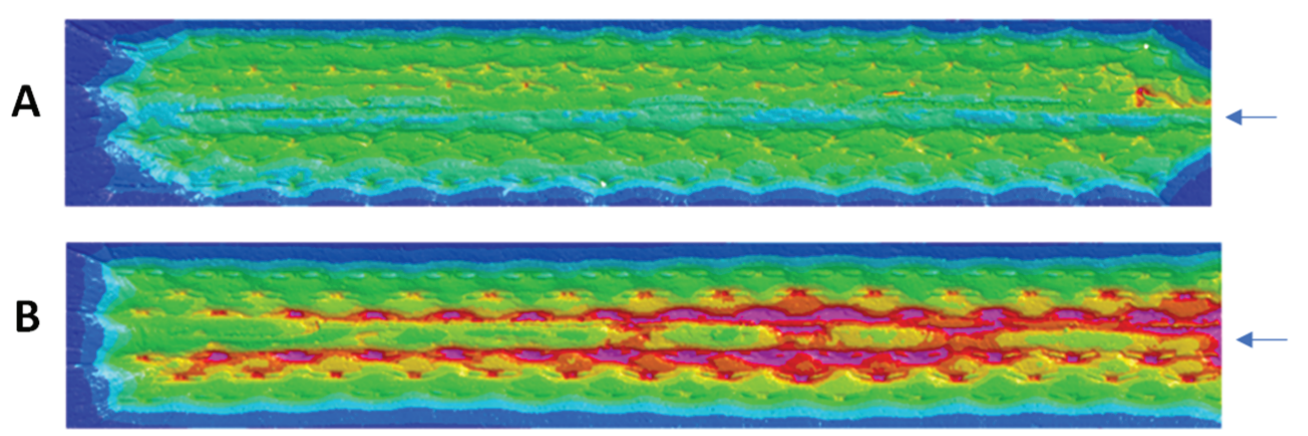

Distal

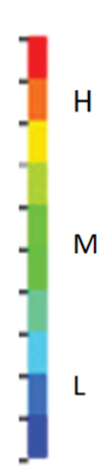

Proximal

Figure 2: Staple line Compression maps for EPS with blue cartridge reload (A), and EGIA using a purple reload (B). Color scale indicates areas of Low (L), Medium (M), and High (H) Pressure. Arrows denote the level of cutlines, with 3 staple rows on either side of the cut line. Distal and proximal ends of staple lines are indicated as shown.

Table 3: Compression force variation across the staple-line (median percent difference between inner and outer staple rows) for EPS green and blue reloads, Signia, and EGIA with purple reloads.

\begin{tabular}{|l|c|c|c|c|}
\hline \multicolumn{1}{|c|}{ Device/reloads } & $\begin{array}{c}\text { Sample number } \\
\text { (N) }\end{array}$ & $\begin{array}{c}\text { Median compression } \\
\text { (Psi) }\end{array}$ & $\begin{array}{c}\text { Median \% difference } \\
\text { (inner vs. outer staples) }\end{array}$ & P- Value $^{2}$ vs. EPS \\
\hline EPS/green and blue & 144 & $\begin{array}{c}\text { Inner (10.037) } \\
\text { Outer (6.669) }\end{array}$ & $39.95 \%$ & N/A \\
\hline Signia/purple & 72 & $\begin{array}{c}\text { Inner (17.389) } \\
\text { Outer (9.383) }\end{array}$ & $62.28 \%$ & $<0.001$ \\
\hline EGIA/purple & 72 & $\begin{array}{c}\text { Inner (16.912) } \\
\text { Outer (9.287) }\end{array}$ & $57.84 \%$ & $<0.001$ \\
\hline Signia and EGIA combined/purple & 144 & $\begin{array}{c}\text { Inner (17.076) } \\
\text { Outer (9.357) }\end{array}$ & $58.92 \%$ & $<0.001$ \\
\hline
\end{tabular}

${ }^{1}$ percent difference $=[$ Inner Row-Outer Row $\div$ Inner Row-Outer Row/2] $\times 100$.

${ }^{2} \mathrm{P}$-values determined by upper-tailed Mann-Whitney test comparing \% difference medians.

Table 4: Tissue transection assessment questionnaire and responses.

\begin{tabular}{|l|c|c|c|}
\hline \multicolumn{1}{|c|}{ Performance Parameter } & Survey Questions & \multicolumn{2}{c|}{ Surgeon response (n) } \\
\cline { 2 - 4 } & & Acceptable & Unacceptable \\
\hline Time required to perform transection(s) & How would you rate the time it takes to complete the \\
transection? & 27 & 0 \\
\hline $\begin{array}{l}\text { Feedback indicating tissue is sealed and } \\
\text { divided as expected }\end{array}$ & $\begin{array}{c}\text { Was the audible and/or tactile feedback throughout the } \\
\text { process acceptable? }\end{array}$ & 27 \\
\hline
\end{tabular}

significantly more uniformity of pressures across the width and length of the staple lines for EPS. In addition, the timing of tissue transections with the EPS stapler as well as adequate tactile and/or audio feedback was acceptable by surgeons, following testing of the device in simulated procedural models.

In view of the factors associated with creation of secure staple lines, such as adequate precompression and firing times [7-9], several design features were incorporated into the new generation EPS stapler to further enhance performance of the device. Dynamic firing allows the EPS motor to slow the firing speed when the device engages thicker, more challenging tissues. Other changes include a more refined anvil curvature as well as wider, tapered staple pockets that are designed to improve the capture and formation of staples when the device is fired. In conjunction with dynamic/slower firing, the newly designed anvil likely contributes toward more uniform compression, fewer malformed staples and fewer staple line leaks as seen in this study. Similar to the new EPS stapler, the current generation ECHELON Powered Plus Stapler also uses gripping surface technology, which was associated with less staple line interventions and fewer hemostatic complications relative to comparator stapler reloads in laparoscopic bariatric surgery [3,11]. In addition, the Echelon stapler was effective for creation of side-to-side laparoscopic intracorporeal anastomoses during emergency colon resections [15]. In this application, precompression times of 20 to 30 seconds were used for adequate tissue compression 
and formation of secure anastomotic lines [15]. Although the current study was limited to benchtop testing, it is anticipated that the EPS may also show favorable performance when evaluated in a clinical setting.

A limitation of this study was the lack of an in vivo assessment of staple line leakage and hemostasis outcomes with EPS, to compare with the benchtop analyses. Favorable benchtop study results, however, indicate that the design changes for EPS are consistent with promotion of proper staple formation and fewer leaks.

\section{Conclusion}

The ECHELON+ Stapler with GST Reloads was shown to provide favorable performance compared to similar competitor stapling systems in benchtop analyses. Future clinical studies will confirm whether the design refinements will translate to enhanced performance in surgical use.

\section{References}

1. Baker RS, Foote J, Kemmeter P, Brady R, Vroegop T, et al. (2004) The Science of Stapling and Leaks. Obes Surg 14: 1290-1298.

2. Chekan E, Whelan RL (2014) Surgical Stapling Device-Tissue Interactions: What Surgeons need to Know to Improve Patient Outcomes. Med Devices (Auckl) 7: 305-318.

3. Fegelman E, Knippenberg S, Schwiers M, Stefanidis D, Gersin KS, et al. (2017) Evaluation of a Powered Stapler System with Gripping Surface Technology on Surgical Interventions Required During Laparoscopic Sleeve Gastrectomy. J Laparoendosc Adv Surg Tech A 27: 489-494.

4. Kimura M, Tanaka H, Hato M, Taniwaki S, Shibata Y, et al. (2016) Evaluation of a New Stapler with Unique Surface Gripping Technology. Br J Med Med Res 18: 1-6.

5. Thompson SE, Young MT, Lewis MT, Boronyak SM, Clymer JW, et al. (2018) Initial Assessment of Mucosal Capture and Leak Pressure After Gastrointestinal Stapling in a Porcine Model. Obes Surg 28: 3446-3453.
6. Collopy BT (2001) Colorectal Anastomotic Leak Rates are Measures of Technical Skill in Surgery. ANZ J Surg 71: 508-510.

7. Matsuzawa F, Homma S, Yoshida T, Konishi Y, Shibasaki S, et al. (2017) Serosal Laceration During Firing of Powered Linear Stapler Is a Predictor of Staple Malformation. Surg Innov 24: 590-597.

8. Nakayama S, Hasegawa S, Hida K, Kawada K, Sakai Y (2015) Obtaining Secure stapling of a Double Stapling Anastomosis. J Surg Res 193: 652-657.

9. Nakayama S, Hasegawa S, Nagayama S, Kato S, Hida K, et al. (2011) The Importance of Precompression Time for Secure Stapling with a Linear Stapler. Surg Endosc 25: 2382-2386.

10. Huang R, Gagner MA (2015) Thickness Calibration Device is Needed to Determine Staple Height and Avoid Leaks in Laparoscopic Sleeve Gastrectomy. Obes Surg 25: 2360-2367.

11. Rawlins L, Johnson BH, Johnston SS, Elangovanraaj N, Bhandari M, et al. (2020) Comparative Effectiveness Assessment of Two Powered Surgical Stapling Platforms in Laparoscopic Sleeve Gastrectomy: A Retrospective Matched Study. Med Devices (Auckl) 13: 195-204.

12. Miller DL, Roy S, Kassis ES, Yadalam S, Ramisetti S, et al. (2018) Impact of Powered and Tissue-Specific Endoscopic Stapling Technology on Clinical and Economic Outcomes of Video-Assisted Thoracic Surgery Lobectomy Procedures: A Retrospective, Observational Study. Adv Ther 35: 707-723.

13. Roy S, Yoo A, Yadalam S, Fegelman EJ, Kalsekar I, et al. (2017) Comparison of Economic and Clinical Outcomes Between Patients Undergoing Laparoscopic Bariatric Surgery with Powered versus Manual Endoscopic Surgical Staplers. J Med Econ 20: 423-433.

14. Rojatkar P, Henderson CE, Hall S, Jenkins SA, Paulin-Curlee GG, et al. (2017) A Novel Powered Circular Stapler Designed for Creating Secure Anastomoses. Glob Surg 4: 94-100.

15. Di Saverio S, Birindelli A, Mandrioli M, Podda M, Binda GA (2017) Intracorporeal anastomoses in emergency laparoscopic colorectal surgery from a series of 59 cases: where and how to do it-a technical note and video. Colorectal Dis 19: 0103-0107. 